\title{
Gender affects immune responses to viruses and vaccines
}

\author{
W. Jean Dodds* \\ DVM; Hemopet, 938 Stanford Street, Santa Monica, CA 90403, USA
}

\begin{abstract}
Gender differences in humans and animals reach beyond their physical features and include the physiological and metabolic traits that reflect immune function, as well as the susceptibility and resistance to disease. Females are predisposed because of their higher estrogen content and their differential responses can include the innate, humoral, and cellular immune systems. Thus, appropriately created and implemented preventive vaccines and immunologically targeted therapies that take both sexes into account should be adopted.
\end{abstract}

Abbreviations: B-Cells: Bursal-Dependent Lymphocytic Cells; HIV: Human Immunodeficiency Virus; IFN: Interferon; NIH: National Institutes of Health; SARS: Severe Acute Respiratory Syndrome; T-Cells: Thymus-Dependent Lymphocytic Cells; TLR7: Toll-Like Receptor-7; WHO: World Health Organization.

\section{Introduction}

A vital function of the immune system is to distinguish between self and non-self antigens, which serves as a specific defense against invading microorganisms, such as viruses, and regulates the body's response to vaccines. Failure of this so-called self-tolerance leads to "autoimmunity", which literally means immunity against self, and can be a consequence of pathophysiological responses to infectious agents and vaccines [1]. is caused by an immune-mediated reaction to selfantigens [1]. Host susceptibility to these adverse events pathological autoimmune states has a genetic basis in humans and animals, is often gender related, and although numerous viruses, bacteria, chemicals, toxins and drugs have been implicated as the triggering environmental agents [1-5]. Females of the species are estimated to be 3-9 times more susceptible than males $[1,2,6]$. The mechanisms involved operate by a process of molecular mimicry and/or non-specific inflammation, and are most often mediated by T-lymphocytes (T-cells) or T-cell dysfunction. The resultant autoimmune diseases reflect the sum of the genetic, gender, and environmental factors involved. As stated in a landmark review "perhaps the biggest challenge in the future will be the search for the environmental events that trigger self-reactivity" [1].

The four main causative factors of autoimmune disease have been stated to be $[1,2]$ :

- Genetic predisposition;

- Hormonal influences, especially of sex hormones;

- Infections, especially of viruses; and

- Stress.

The following discussion summarizes these points.

\section{Discussion}

The accumulated evidence from research studies with experimental animal models and clinical experience in human and animal patients has pointed to the genetic and environmental factors involved in promoting autoimmune disorders [1,2,7-9].

\section{Genetic predisposition}

Genetic and genomic factors that predispose to autoimmune disorders in humans have been linked to the leucocyte antigen D-related gene locus of the major histocompatibility complex, and parallel associations are seen in domestic animals [3,7-9]. Inbred and line bred families of purebred dogs, for example, are at increased disease risk [2-4,7].

\section{Immune-suppressant viruses and vaccines}

Viral diseases in humans and companion animals are increasingly recognized contributors to systemic immune-mediated disorders affecting a wide variety of tissues [5,7-14]. Immune-suppressant viruses of the retrovirus and parvovirus classes have been implicated as causes of bone marrow failure, immune-mediated blood diseases, hematologic malignancies (lymphoma and leukemia), dysregulation of humoral and cell-mediated immunity, organ dysfunction and failure (liver, kidney), and autoimmune endocrine disorders especially of the thyroid and adrenal glands, and pancreas [5,7-13]. Recent vaccination of humans and companion animals within the prior 30-45 days is also recognized as an equal contributor to these disorders [10-14].

\section{Gender differences}

Sex differences of humans and animals extend beyond their anatomical physical features to the physiological and metabolic traits that affect important immune system functions, thereby predisposing males and females to respond differently to infectious diseases, especially of the viral type [6]. As an example, estrogens tend to promote stronger inflammatory, cellular, and humoral immune responses in females than in males [15-18]. Strong immune responses to pathogens can benefit

Correspondence to: W. Jean Dodds, DVM; Hemopet, 938 Stanford Street, Santa Monica, CA 90403, USA, Tel: 714-891-2022 Ext 115; E-mail: info@hemopet.org

Key words: immune responses, gender, vaccines

Received: November 08, 2016; Accepted: December 05, 2016; Published: December 08, 2016 
the host individual, whereas overactive inflammatory responses can damage host tissues. These differences predispose females more than males to develop immune dysfunction and pathologies, comorbidities, autoimmune disorders, and adverse reactions to vaccines, among other deleterious effects $[6,15-21]$.

\section{Gender-related responses to and side effects of vaccines}

The gender differences in immune responses to viral antigens also apply to vaccines, as has been recognized in humans and animals for some time, and shown by the variability in vaccine efficacy between males and females [6,12-14,19]. Humoral immune responses are stronger in women, due to the fact that estradiol stimulates B-lymphocytes (B-cells) to proliferate and produce antibodies [15,16].

This heightened immune reactivity in females versus males benefits them in several ways by better protecting them when vaccinated against a specific viral strain, but also providing more cross-protection from similar viral strains [17]. In clinical trials of herpes simplex vaccine, when data were analyzed by sex, the efficacy of the vaccine was $73 \%$ in women and only $11 \%$ in men [19]. However, when the results for both sexes were combined, the aggregate data results masked how well the vaccine protects women from herpes simplex viral infection [6]. Use of this vaccine has not been promoted or encouraged, which is especially tragic, because it could not only protect women against this sexually transmitted disease but also could protect their partners during intimate contact [6].

\section{Gender-related inflammatory responses to influenza virus}

Morbidity and mortality are often higher for women during influenza out breaks and pandemics, [6]. The cumulative evidence from the outbreaks ranging from 1957-2009 caused the World Health Organization (WHO) in 2010 to urge that researchers and epidemiologists consider sex differences in evaluating exposures to the influenza viruses and the outcomes [6].

Male mice tolerate much higher doses of influenza virus than do females; the lethal dose of the H1N1 influenza virus is 11-fold higher for males than comparable female mice [17]. Host-mediated immunopathology, rather than virus replication itself, underlies the sex differences in influenza pathogenesis, such that female mice exhibit a heightened "cytokine storm" that results in greater weight loss, hypothermia, and earlier deaths than in males [17].

Women generate nearly twice the serum antibody titers after being vaccinated against the influenza virus than do men; and equivalent antibody titers to men are achieved when they are given just half of the influenza vaccine dose [19]. Females also mount stronger cellular and innate immune responses to vaccines. However, the overactive immune responses of women can predispose them to more frequent and severe adverse side effects than men who receive the same vaccine. Side effects such as localized inflammation, headache, fever, nausea, rash, and fatigue are more common in women than men for both viral and bacterial vaccines $[6,15]$.

Sex hormones play a leading role in predisposing females to immunopathology from influenza and other viral infections [6]. Sex steroids, like estradiol, alter the functioning of immune cells by binding to specific receptors expressed in a variety of lymphoid tissue cells, including circulating lymphocytes, macrophages and dendritic cells. When estradiol levels are high, they act to signal protection whereby these inflammatory tissue responses are blocked [15,16]. Estradiol acts as a signaling biomarker for protection from this disease. By contrast, during an influenza infection, estradiol levels drop and so inflammatory responses are boosted. Restoring estradiol in infected mice causes a more than 10-fold blocking of inflammatory cell recruitment, thereby significantly protecting females by suppressing inflammatory cytokines and chemokines, reducing morbidity and mortality following H1N1 infection. Progesterone can also protect female mice from immunopathology by promoting epithelial cell proliferation in the lungs to help heal inflammatory damage $[15,16]$.

\section{Gender-related effects of HIV accelerate disease and comorbidities in women}

Men and women also respond differently when infected with human immunodeficiency virus (HIV) [20,21]. Due to heightened Toll-like receptor-7 (TLR7) responses, women tend to control HIV better than men. These strong antiviral responses in women keep the virus under control in early HIV infection, keeping their viral loads about $40 \%$ lower than those in men [6].

However, although HIV replication is initially controlled more effectively in women than men, chronic HIV disease progresses faster and can lead to earlier comorbidities when elevated inflammation and other host immune responses lead to collateral damage. In addition, chronically heightened inflammatory responses can damage other tissues, leading to non-HIV related comorbidities, cardiovascular disease, and premature aging [21].

The hyperactive immune responses in females can be detrimental against some viral infections but beneficial against others [18]. Although heightened TLR-7 responses in females contribute to influenza-triggered immunopathology, such responses can protect women against coronaviruses, like those causing severe acute respiratory syndrome (SARS) -- first identified in Asia in 2003 -- by helping to clear the viruses more quickly [18]. Similarly, female mice control coronavirus infections better than do their male counterparts, and being female profoundly affects the production of interferon (IFN) type 1 which promotes viral clearance [18]. Among humans, men appear to have higher fatality rates than do women following infections with coronaviruses such as SARS [6].

The so-called "immune braking" mechanisms, discussed above, also contribute to noninfectious diseases, such as autoimmunity and cancers. When malfunctioning, CD200, a modulator of TLR-7 activity, can dampen these responses [18]. The opposite effect could occur, however, as this regulator may predispose women to developing the autoimmune disorder, systemic lupus erythematosus, as TLR-7 responses are intrinsically increased in women. Clinically, women with lupus outnumber men 9 to 1 [18].

In humans with cancer, CD200 signaling is upregulated, damping immune functions that could otherwise identify and eradicate tumors. In particular, multiple myeloma and acute myeloid leukemia patients tend to overexpress the inhibitory CD200 signal, which is associated with a poor prognosis for such patients $[16,18]$. However, CD200 blockers could exert pathological effects on women, elevating their risk of developing autoimmune disorders and increasing the severity of influenza infections [18].

In summary conclusion, these researchers assert that the design of vaccines, their adjuvants, and vaccine strategies should be sex-specific, beginning with infants, to reduce adverse reactions in females and increase immunogenicity in males [6]. 
Generally, sex-specific responses should be taken into account when developing and implementing both prophylactic and treatment strategies for infectious diseases and for noninfectious diseases such as autoimmune diseases, inflammatory conditions, and cancers, for which the immune system responses play important roles [6]. Future vaccines and even drug designs need to exploit the beneficial immunogenic effects while minimizing deleterious effects.

The very broad scope and challenges presented by these gender response differences largely account for the failure to recognize and effectively address them. A Lastly, one significant obstacle to overcome in this regard is the tendency of researchers, health care clinicians, and regulatory officials to protect the status quo. The prevailing approach is to deny or avoid the fact that gender--related differences exist and are important considerations in determining not only susceptibility to diseases and vaccine efficacy but also their risk outcomes [6].

\section{Role of stress events}

A final and equally important role in disease prevention, susceptibility and management revolves around the effects of both "good" (beneficial) and "bad" (harmful) stress [2,22]. The purpose of a physiological stress response is adaptive, to allow for survival during flight or fight circumstances. While long-term stress is generally harmful, short-term stress can be protective as it helps prepare the individual to deal with immune challenges [22]. This immune biological stress response can be triggered by psychological, physiological, or physical (exercise, trauma) stressors. The goal of promoting health and healing to enhance individual well-being is to enhance the effects of "good" stress while minimizing the effects of "bad" stress [22].

\section{Conclusion}

Evidence of gender differences in the immune responses of both healthy and diseased humans and animals has been known for many years. This is particularly relevant to the body's responses to viruses and vaccines. Implementing the appropriate gender-related paradigm shifts in the diagnosis, management and treatment of infectious and vaccine immunological effects is long overdue.

\section{References}

1. Sinha AA, Lopez MT, McDevitt HO (1990) Autoimmune diseases: the failure of selftolerance. Science 248: 1380-1388. [Crossref]

2. Dodds WJ (2002) Complementary and alternative veterinary medicine: the immune system. Clin Tech Small Anim Pract 17: 58-63. [Crossref]
3. Dodds WJ (1992) Genetically based immune disorders: Autoimmune diseases, Parts 1-3. Vet Pract STAFF, 4 (1, 2, and 3): 8-10, 1, 26-31, 35-37.

4. Dodds WJ (1992) Immune deficiency diseases: Genetically based immune disorders, Part 4. Vet Pract STAFF, 4 (5): 19-21.

5. Rosenfeld SJ, Young NS (1991) Viruses and bone marrow failure. Blood Rev 5: 71-77. [Crossref]

6. Weiman S (2016) Immune responses to viruses and vaccines differ between men and women. Microbe 11: 383-387.

7. Happ GM (1995) Thyroiditis-a model canine autoimmune disease. Adv Vet Sci Comp Med 39: 97-139. [Crossref]

8. Tomer Y, Davies TF (1993) Infection, thyroid disease, and autoimmunity. Endocr Rev 14: 107-120. [Crossref]

9. Schmidt MA, Bland JS (1997) Thyroid gland as sentinel: Interface between internal and external environment. Altern Ther 3: 78-81. [Crossref]

10. Cohen AD, Shoenfeld Y (1996) Vaccine-induced autoimmunity. J Autoimmun 9: 699703. [Crossref]

11. Dodds WJ (1995) Estimating disease prevalence with health surveys and genetic screening. Adv Vet Sci Comp Med 39: 29-96. [Crossref]

12. Dodds WJ (1997) Vaccine-related issues. In Complementary and Alternative Veterinary Medicine, eds. AM Schoen, SG Wynn, 40:701-712. Mosby, NY.

13. Dodds WJ (1999) More bumps on the vaccine road. Adv Vet Med 41: 715-732 [Crossref]

14. Tizard I, Ni Y (1998) Use of serologic testing to assess immune status of companion animals. J Am Vet Med Assoc 213: 54-60. [Crossref]

15. Robinson DP, Hall OA, Nilles TL, Bream JH, Klein SL (2014) 17ß-estradiol protects females against influenza by recruiting neutrophils and increasing virus-specific CD8 T cell responses in the lungs. $J$ Virol 88: 4711-4720.

16. Robinson DP, Lorenzo M, Jian W, Klein SL (2011) Elevated 17ß-estradiol protects females from influenza A virus pathogenesis by suppressing inflammatory responses. PLoS Pathogens 7: e1002149.

17. Lorenzo M, Hodgson A, Kaplan J, Robinson DP, Pekosz A, et al., (2011) Antibody responses and cross protection against lethal influenza A viruses differ between the sexes in C57BL/6 mice. Vaccine 29:9246-9255. [Crossref]

18. Karnam G, Rygiel TP, Raaben M, Grinwis GC, Coenjaerts FE, et al., (2012) CD200 receptor controls sex-specific TLR7 responses to viral infection. PLoS Pathog 8: e1002710. [Crossref]

19. Klein SL, Jedlicka A, Pekosz A (2010) The Xs and Y of immune responses to viral vaccines. Lancet Infect Dis 10: 338-349. [Crossref]

20. Griesbeck M, Scully E, Altfeld M (2016) Sex and gender differences in HIV-1 infection. Clin Sci (Lond) 130: 1435-1451. [Crossref]

21. Hagen S, Altfeld M (2016) The X awakens: multifactorial ramifications of sex-specific differences in HIV-1 infection. $J$ Virus Erad 2: 78-81. [Crossref]

22. Dhabhar FS (2014) Effects of stress on immune function: the good, the bad, and the beautiful. Immunol Res 58: 193-210. [Crossref]

Copyright: (C2016 Jean Dodds W. This is an open-access article distributed under the terms of the Creative Commons Attribution License, which permits unrestricted use, distribution, and reproduction in any medium, provided the original author and source are credited. 